\title{
Ansiedad matemática y desempeño académico en estudiantes en la formación básica de ingeniería
}

\author{
Myriam Ortiz-Padilla ${ }^{(1)}$, Martha Paredes-Bermúdez ${ }^{(2)}$, Roberto Soto-Varela ${ }^{(3)}$ y Edna Aldana-Rivera ${ }^{(4)}$ \\ (1) Facultad de Ciencias Jurídicas y Sociales, Universidad Simón Bolívar, Barranquilla-Colombia. \\ (correo-e: mortiz@unisimonbolivar.edu.co) \\ (2) Centro de Investigaciones, Instituto Nacional de Formación Técnica Profesional "Humberto Velásquez García, \\ Ciénaga-Magdalena -Colombia, (correo-e: paredesbermudez1961@gmail.com) \\ (3) Facultad de Educación y Letras, Universidad Nebrija, Madrid-España. (correo-e: rsoto@nebrija.es) \\ (4) Facultad de Ciencias de la Salud, Universidad Simón Bolívar, Barranquilla-Colombia. \\ (correo-e: ealdana@unisimonbolivar.edu.co)
}

Recibido Nov. 26, 2019; Aceptado Ene. 23, 2020; Versión final Mar. 16, 2020, Publicado Ago. 2020

\begin{abstract}
Resumen
El objetivo del estudio es analizar la relación entre la ansiedad matemática percibida y el rendimiento académico en estudiantes de Ingeniería de una universidad privada de Barranquilla-Colombia. La metodología fue de corte cuantitativo y un diseño descriptivo-correlacional. El instrumento utilizado fue la Escala Abreviada de Ansiedad Matemática (AMAS), en su adaptación al español. Para el rendimiento académico se tomaron los datos del sistema de información de la institución y categorizaron según normatividad vigente. La muestra intencional correspondió a 87 estudiantes. Los resultados no evidencian relación existente entre la ansiedad matemática y el desempeño académico, en cambio sí se identifican relaciones entre la edad y la ingeniería que estudian, y entre la ansiedad matemática global y la ingeniería. Se proponen otras variables relacionadas para explicar un problema complejo como el aprendizaje de las matemáticas. En conclusión, los resultados descriptivos muestran que los estudiantes presentan en un alto porcentaje de ansiedad moderada.
\end{abstract}

Palabras clave: ansiedad matemática; desempeño académico; formación universitaria; formación de ingenieros

\section{Mathematical anxiety and academic performance in engineering students}

\begin{abstract}
The objective of the study is to analyze the relationship that exists between perceived mathematical anxiety and academic performance in engineering students at a private university in Barranquilla, Colombia. The methodology was quantitative and with a descriptive-correlational design. The instrument used was the Abbreviated Mathematical Anxiety Scale (AMAS), in its Spanish adaptation. Academic performance was based on data from the Institution's Information System and was categorized according to current regulations. The sample was 87 students. The results do not evidence a relationship between mathematical anxiety and academic performance. Instead, the results show relationships between student age and engineering subject and between global mathematical anxiety and engineering. This study proposes other related variables to explain the complex problem of learning mathematics. In conclusion, the descriptive results suggest that there is a high percentage of moderate anxiety among students.
\end{abstract}




\section{INTRODUCCIÓN}

En la formación de ingenieros resulta imprescindible el uso de las matemáticas para resolver situaciones y utilizar un pensamiento sistemático y reflexivo, siendo consideradas estas como una de las principales herramientas que favorecerá su desempeño profesional posterior. El pensamiento matemático y la alfabetización cuantitativa, son considerados como competencias genéricas (Maury et al., 2018). Sin embargo, hay una multitud de factores que juegan un papel importante en el aprendizaje de las matemáticas en los jóvenes estudiantes de ingeniería de las universidades: factores sociales, cognitivos, culturales, emocionales e incluso la disponibilidad de utilizar léxico en el ámbito de datos y azar y los esquemas de razonamiento formal (Gamal et al., 2017). Dentro de los factores emocional-afectivos, la ansiedad hacia las matemáticas -AM, se vuelve muy importante. Esta ansiedad se define como aquellos "sentimientos desagradables de tensión y ansiedad que dificultan la capacidad de lidiar con los números y las matemáticas en una variedad de situaciones" (O'Leary et al., 2017, p.1) y que involucra tres tipos de componentes: afectivos, cognitivos y conductuales (García-Santillán et al., 2018). Esta forma de ansiedad se define entonces muy específicamente asociada al aprendizaje de contenidos matemáticos. Entre los componentes conductuales a los que lleva lugar este tipo de emoción están los comportamientos inadecuados, la inasistencia a clase y a evitar los cursos de matemáticas, entre otros.

En cuanto a los aspectos cognitivos asociados, se encuentran los pensamientos intrusivos e inhibitorios que pueden surgir en el individuo de forma disruptiva e involuntaria con contenidos de desesperanza, preocupación, miedo al fracaso, asociados por lo tanto a emociones negativas. Este tipo de pensamientos que se apoderan de la conciencia causan una disminución en la capacidad de la memoria de trabajo que tiene entonces que ocuparse en gran medida de resolverlos disminuyendo la eficacia y eficiencia de la tarea matemática (Justicia-Galiano et al., 2016).

Los efectos de la ansiedad van desde síntomas físicos ya investigados, hasta la afectación del rendimiento de los estudiantes en las clases, evaluaciones, exámenes estandarizados e incluso sus decisiones sobre las trayectorias profesionales a seguir (Maloney et al., 2013). Además, estos sentimientos influyen negativamente en el autoconcepto del alumno, lo que afecta su confianza en el aprendizaje de ésta área de conocimiento. En un estudio realizado por Agüero et al. (2017), demuestra que los alumnos con más ansiedad hacia las matemáticas desarrollan menor confianza, creen poco en sus habilidades para enfrentarse a las tareas matemáticas y se sienten con menos habilidad a la hora de enfrentarse a ella, con todos los aspectos negativos que eso desencadena.

En el campo de la educación matemática, la AM se ha vuelto en un elemento que se está tomando muy en cuenta, a la hora de analizar las distintas razones por las cuales los alumnos tienen dificultades a la hora de entender ciertos constructos, los algoritmos de las operaciones, la resolución de problemas, con las matemáticas en general, independientemente del nivel educativo al que nos refiramos, ya que es un problema que afecta de forma transversal a todos ellos. Cerda et al. (2016) indican que ciertas emociones se activan al momento de aprender y que juegan un papel muy importante en el desarrollo de las tareas cognitivas requeridas. Así emociones positivas como el interés, la curiosidad, la alegría disponen al logro y emociones como la ansiedad, la desesperanza, el miedo pueden provocar bloqueo de estos procesos y llevar a un fracaso o desajuste en los procesos académicos ya que no permiten el procesamiento fluido de la información. En este sentido, se deben realizar esfuerzos para conocer su comportamiento en cada región, país, institución educativa, a fin de poder desarrollar acciones relevantes que mejoren el desempeño del estudiante en matemáticas y otras disciplinas cuantitativas, puesto que el componente social, influye en cómo se enseñan y aprenden las matemáticas, pese a que guardan aspectos comunes entre ellos, como son la enseñanza de los algoritmos de las operaciones algebraicas o el lenguaje propio matemático. (Eccius-Wellmann et al., 2017).

Estudios llevados a cabo por Isiksal et al. (2009) o Jansen et al. (2013), indican una relación inversa entre la ansiedad matemática y el rendimiento matemático; no obstante, no hay un consenso unánime al respecto, ya que otro estudio conducido por Krizinger et al. (2009), no ve relación entre las variables mencionadas.

A su vez Carey et al. (2016) indican una relación bidireccional entre ellos, lo que significa que la ansiedad matemática y el rendimiento académico pueden influirse mutuamente y convertirse en un círculo vicioso difícil de romper, o en palabras de Ashcraft y Ridley (2005) la ansiedad que se puede presentar hacia las matemáticas, provoca un malestar y sobretodo, conductas reticentes, incluso evasivas que incide en una disminución de calidad y cantidad que los estudiantes se exponen a esta área de conocimiento, lo que causa un peor aprendizaje y, por lo tanto, un peor rendimiento; y a su vez como se ha comentado, ese peor rendimiento parece generar una mayor ansiedad ante la realización de tareas matemáticas, favoreciendo estas conductas evitativas. 
En la relación de ansiedad matemática y género, los estudios concluyentes no son reconocidos, puesto que Pérez-Tyteca et al. (2011), argumentan que las mujeres tienden a sufrir más ansiedad matemática que los hombres al mostrar más síntomas físicos (como nervios y tensión entre otros); por lo que tiende a haber un menor rendimiento matemático en esta área de parte de ellas, lo que conduciría a evitar los cursos cuantitativos y limita su educación futura y opciones de carrera. García-Santillán et al. (2018), en la línea del anterior, indican que las mujeres pese a que los hombres y mujeres presentan igual capacidad cognitiva, son estas las que obtienen peores resultados en sus exámenes, debido a que su rendimiento a estas pruebas, se ve comprometido por la presencia de la ansiedad. Como nos encontramos en el caso de México, donde los varones llegan a obtener hasta siete puntos más de media en cuanto a su rendimiento académico, ya que el factor ansiedad es menos lesivo y les afecta menos a la hora de la realización de las pruebas que miden su desempeño.

El reconocimiento de la ansiedad matemática ha aumentado, incluido su valor en pruebas internacionales, como PISA, que identifica que los estudiantes que se sienten ansiosos no están interesados en sus estudios, tienen un peor rendimiento y tienen menos confianza en sus habilidades para enfrentar problemas matemáticos (Pérez-Tyteca et al., 2013). Por ejemplo, la investigación realizada con el rendimiento matemático de los estudiantes españoles en las pruebas PISA 2012 reveló que el riesgo de bajo rendimiento matemático varía según el nivel de ansiedad matemática presente. Además, se reconoce que se requiere un nivel de ansiedad matemática para movilizar al alumno para que sea eficiente. Si se excede, este nivel puede tener un efecto negativo en su rendimiento matemático (Bauselas-Herreras, 2018).

En cuanto al rendimiento en matemáticas de los estudiantes colombianos en pruebas internacionales como PISA, en 2018, Colombia ocupó el lugar 70 entre 79 países participantes con un puntaje promedio de 391 (OCDE, 2019). Estos resultados no fueron diferentes en comparación con los años de evaluación previos, solo mejorando en 1 punto con respecto a la anterior edición de PISA, evaluada en 2015 (ICFES, 2017). Reali et al. (2016), señalan que hay muchos factores que pueden estar generando este bajo rendimiento en los estudiantes colombianos (por ejemplo, grandes desafíos que enfrenta el sistema educativo relacionado con la contratación de docentes, el sujeto de la evaluación, y las posibilidades de mejora en la educación matemática), pero estos autores indicaron que la ansiedad matemática podría ser un factor importante que influye en los puntajes de la prueba PISA.

Es entonces relevante reconocer en los estudiantes este fenómeno para así poder desarrollar acciones para mejorar el desempeño matemático y la creación de entornos educativos más efectivos que garanticen el crecimiento del potencial de todos los estudiantes en esta importante área (Eccius-Wellmann et al., 2017; Maloney et al., 2013). A partir de ello, esta investigación se propone describir en primera instancia como se presenta la ansiedad matemática y el rendimiento académico de los estudiantes de distintos grados de ingeniería de una universidad privada en Barranquilla-Colombia para de manera posterior poder analizar las posibles relaciones entre estas dos variables y otras asociadas.

\section{METODOLOGIA}

El estudio es de corte cuantitativo y un diseño descriptivo correlacional, en el que se pretende describir cada una de las variables para posteriormente encontrar posibles relaciones entre ellas. 1) Participantes: Formaron parte del estudio 87 estudiantes de los programas de ingeniería ofrecidos por una universidad privada de Barranquilla Colombia: Ingenieria Industrial, Ingeniería de mercados e Ingeniería de Sistemas, que se encontraban cursando de I a II semestre en el 2017-2. El 54, 5\% estaban en el grupo de 15 a 17 años; seguido del grupo de 18 a 22 años con un 37,5\%, con predominio del género masculino en un 65,9\%; y una mayor participación del programa de ingeniería industrial con un $43,2 \%$, seguido de ingeniería de sistemas con un $36,4 \%$. Los estudiantes que participaron firmaron voluntariamente el consentimiento informado. 2) Materiales: Para la recogida de datos se utilizó la Escala Abreviada de Ansiedad Matemática (Abbreviated Math Anxiety Scale- AMAS). Este cuestionario breve, desarrollado por Hopko et al. (2003), evalúa la ansiedad a las matemáticas en dos dimensiones: Ansiedad hacia el aprendizaje y la Ansiedad hacia la evaluación. Está compuesto por 9 cuestiones en las que los sujetos deben decidir cuanta ansiedad les provocan diferentes situaciones relacionadas con las matemáticas.

En esta investigación se utilizó la adaptación al español realizada por Brown y Macías (2016). Para el rendimiento académico los datos se obtuvieron del Sistema de información de la institución. 3) Análisis de datos: Los datos obtenidos a través de la aplicación de la escala fueron sometidos a un análisis descriptivo e inferencial, para ello se empleó el software estadístico SPSS, versión 20. El análisis descriptivo, llevado a cabo en la primera parte del estudio, permitió la representación gráfica de las variables mediante tablas de distribución de frecuencias y porcentajes. El análisis inferencial se utilizó con el objetivo de contrastar las hipótesis planteadas. De acuerdo al tipo de variable se utilizaron las siguientes pruebas no paramétricas: prueba de Kolmogrov-Smirnov, Coeficiente de correlación de Spearman y Prueba de Kruskal-Wallis. 


\section{RESULTADOS}

En primera instancia se presentan los resultados del análisis descriptivo general de cada una de las variables de estudio para el caso de la AM se identifican los niveles en que se manifiesta esta emoción en los estudiantes primero de manera general y posteriormente en cada una de sus dimensiones. Estos resultados se relacionan con variables como la edad, género y tipo de ingeniería. Posteriormente se hace el análisis que relaciona el nivel de AM de los estudiantes con el desempeño académico basado en métodos estadísticos.

Tabla 1: AM general por tipo de ingeniería

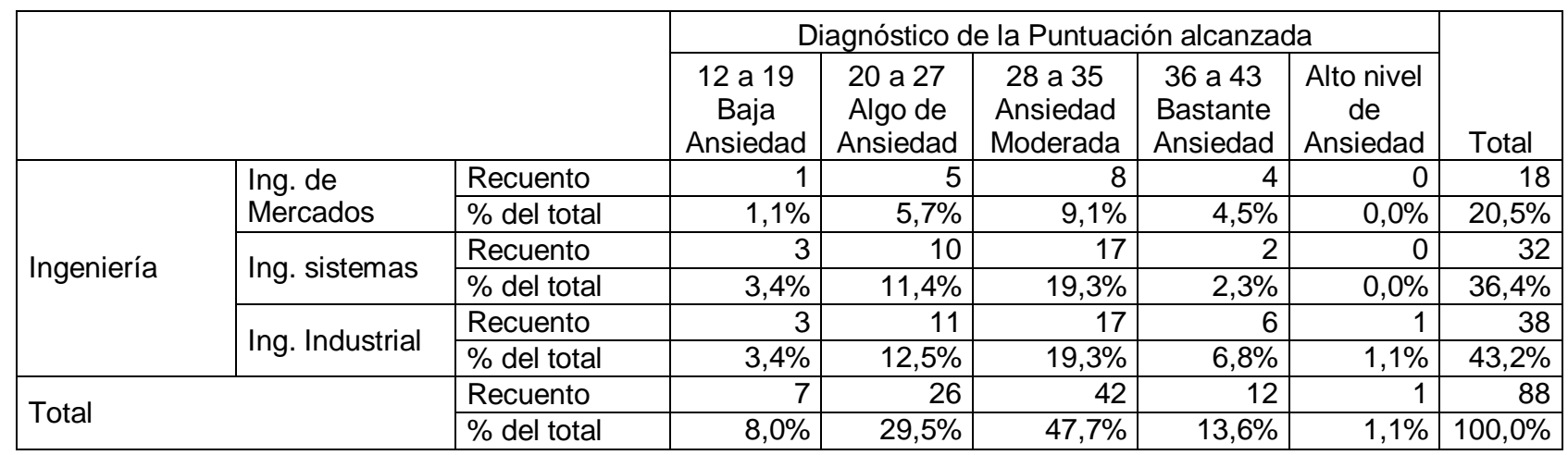

En cuanto a la dimensión de Ansiedad percibida por los estudiantes durante la evaluación los resultados indican niveles mayores, presentando Bastante ansiedad en un $42 \%(n=37)$, seguido de Alto nivel de ansiedad en el $23.9 \%(n=21)$. Entre las situaciones que generan más ansiedad en los estudiantes percibiendo un nivel de Bastante y Alta ansiedad están en su orden: Sentirse ansioso un día antes de presentar una prueba de matemáticas, Sentir ansiedad al realizar un examen en el curso de matemáticas y en un nivel alto en un $39.8 \%(n=35)$ de los participantes se sienten ansioso cuando en la clase de matemáticas le realizan preguntas.

Al revisar las relaciones de la variable AM con la variable edad se encuentra que en todos los grupos de edad predominó el nivel de Ansiedad Moderada siendo mayor en los jóvenes de 15 a 17 años, seguido del grupo de 18 a 22 años. A medida que la edad va avanzando la tendencia es a disminuir la percepción de ansiedad ante situaciones de aprendizaje y evaluación en matemáticas. También se analizó la presencia de AM según el género, encontrándose que la ansiedad Moderada frente a las matemáticas es mayor en el hombre con un $65,9 \%$., que en la mujer con un $34.1 \%$. No obstante, al observar las medias se observa que las mujeres obtienen ésta en 2.7 puntos, mientras que los hombres obtienen un 2.4 .

En cuanto al nivel de desempeño académico en matemáticas resultado de su proceso durante el semestre cursado en una asignatura con este componente principal se observó las categorías de Aprobados en un $87 \%(n=77)$; como Excelente el $3,4 \%(n=3)$ y el $9.1 \%$ fueron no aprobados. Al relacionar el Nivel de ansiedad frente a las matemáticas obtenido por el AMAS y el Rendimiento según el desempeño de los estudiantes se observó que del $87 \%(n=77)$ aprobados, el $45 \%$ presentaron un nivel de Ansiedad Moderada a nivel general. Para los estudiantes que obtuvieron un desempeño excelente las percepciones frente a AM varían entre los niveles Bajo, Algo y Moderado. Los estudiantes que No aprobaron perciben altos niveles de la categoría Bastante Ansiedad.

Tras reseñar los aspectos más destacados del análisis descriptivo de la muestra, se pasa al análisis estadístico de la misma para poder ser más precisos en dicho análisis. El primer análisis efectuado fue para determinar la fiabilidad del instrumento en cuestión, para ello se usó Alfa de Cronbach. El valor para el cuestionario en su totalidad fue de 0.809 (con una confianza del $95 \%$ intervalo entre $0.78-0.84$ ), para la subescala Ansiedad en el aprendizaje de las matemáticas fue de 0.793 (con una confianza del 95\% intervalo entre 0.76-0.82) y para la subescala Ansiedad en la evaluación de las matemáticas que fue de 0.704 (al 95\% de confianza con un intervalo de 0.67-0.73). El Alfa de Cronbach no se ve incrementado al eliminar ninguno de los ítems del cuestionario, ni al eliminar ninguna de las subescalas del total.

Comprobamos a continuación si la muestra se distribuye de una forma normal o no, para determinar el tipo de análisis a utilizar. Puesto que la muestra es superior a 50 sujetos tal se debe usar el estadístico de Kolmogorov-Smirnov el cual determina que la muestra no se comporta de forma normal ni en el total del cuestionario $\left[K S_{(88)}=0.00, p<0.05\right]$, ni en la subescala Ansiedad matemática [ $K S_{(88)}=0.00, p<0.05$ ] y tampoco para la subescala Ansiedad en la evaluación $\left[K S{ }_{(88)}=0.00, p<0.05\right]$. Se realizó un análisis correlacional a través del Coeficiente de Spearman para poder comprobar cómo se relacionan las distintas variables entre sí. Lo que reflejan los resultados es que existe una correlación moderada negativa entre la variable Edad 
Ingeniería (rho= $-0,424 ; s i g=0.000$ ), a su vez nos encontramos con una correlación positiva entre la Ingeniería y la AM GLOBAL ( $\mathrm{rho}=1,000 ; \mathrm{sig}=0.000$ ); y a su vez arroja una correlación moderada negativa entre la Edad y la AM GLOBAL (rho= -0,424; sig=0.000), tal y como se puede comprobar en la tabla 1 .

A continuación, se realiza un análisis univariado con el propósito de examinar las posibles relaciones existentes de las variables identificadas en la fase anterior y la variable dependiente del modelo. En concordancia a la metodología expuesto por Rodriguez-Ayán (2005), para analizar las asociaciones univariadas se debe utilizar el estadístico Kruskal-Wallis. La tabla número 2., muestra la asociación entre la variable dependiente y las variables independientes del modelo. Dicha tabla muestra una varianza significativa en el comportamiento de la AM GLOBAL en función de la Ingeniería, Ansiedad en el aprendizaje y en la Ansiedad en la evaluación ya que la significación para estas variables es de $p<0.05$.

Tabla 2: Correlación de Spearman entre las variables de estudio. **La correlación es significativa en el nivel 0,01(bilateral)

\begin{tabular}{|c|c|c|c|c|c|c|c|c|}
\hline & & Edad & Sexo & Ingeniería & $\begin{array}{c}\text { Ansiedad } \\
\text { Aprendizaje }\end{array}$ & $\begin{array}{l}\text { Ansiedad } \\
\text { Evaluación }\end{array}$ & $\begin{array}{c}\text { AM } \\
\text { GLOBAL }\end{array}$ & Desempeño \\
\hline Edad & $\begin{array}{l}\text { Coeficiente } \\
\text { de } \\
\text { correlación }\end{array}$ & 1.000 & 0.082 &,$- 424^{\star \star}$ & 0.069 & 0.127 &,$- 424^{\star *}$ & -0.049 \\
\hline & Sig.(bilateral) & & 0.446 & 0.000 & 0.523 & 0.238 & 0.000 & 0.652 \\
\hline & $\mathrm{N}$ & 88 & 88 & 88 & 88 & 88 & 88 & 88 \\
\hline Sexo & $\begin{array}{l}\text { Coeficiente } \\
\text { de } \\
\text { correlación }\end{array}$ & 0.082 & 1.000 & 0.020 & 0.049 & 0.186 & 0.020 & 0.039 \\
\hline & Sig.(bilateral) & 0.446 & & 0.851 & 0.653 & 0.083 & 0.851 & 0.716 \\
\hline & $\mathrm{N}$ & 88 & 88 & 88 & 88 & 88 & 88 & 88 \\
\hline Ingeniería & $\begin{array}{l}\text { Coeficiente } \\
\text { de } \\
\text { correlación }\end{array}$ &,$- 424^{* *}$ & 0.020 & 1.000 & 0.060 & -0.025 & $1.000^{* *}$ & -0.053 \\
\hline & Sig.(bilateral) & 0.000 & 0.851 & & 0.578 & 0.816 & & 0.623 \\
\hline & $\mathrm{N}$ & 88 & 88 & 88 & 88 & 88 & 88 & 88 \\
\hline $\begin{array}{l}\text { Ansiedad } \\
\text { Aprendizaje }\end{array}$ & $\begin{array}{l}\text { Coeficiente } \\
\text { de } \\
\text { correlación }\end{array}$ & 0.069 & 0.049 & 0.060 & 1.000 & 0.151 & 0.060 & -0.041 \\
\hline & Sig.(bilateral) & 0.523 & 0.653 & 0.578 & & 0.161 & 0.578 & 0.706 \\
\hline & $\mathrm{N}$ & 88 & 88 & 88 & 88 & 88 & 88 & 88 \\
\hline $\begin{array}{l}\text { Ansiedad } \\
\text { Evaluación }\end{array}$ & $\begin{array}{l}\text { Coeficiente } \\
\text { de } \\
\text { correlación }\end{array}$ & 0.127 & 0.186 & -0.025 & 0.151 & 1.000 & -0.025 & -0.061 \\
\hline & Sig.(bilateral) & 0.238 & 0.083 & 0.816 & 0.161 & & 0.816 & 0.574 \\
\hline & $\mathrm{N}$ & 88 & 88 & 88 & 88 & 88 & 88 & 88 \\
\hline AM GLOBAL & $\begin{array}{l}\text { Coeficiente } \\
\text { de } \\
\text { correlación }\end{array}$ &,$- 424^{* *}$ & 0.020 & $1,000^{* *}$ & 0.060 & -0.025 & 1.000 & -0.053 \\
\hline & Sig.(bilateral) & 0.000 & 0.851 & & 0.578 & 0.816 & & 0.623 \\
\hline & $\mathrm{N}$ & 88 & 88 & 88 & 88 & 88 & 88 & 88 \\
\hline Desempeño & $\begin{array}{l}\text { Coeficiente } \\
\text { de } \\
\text { correlación }\end{array}$ & -0.049 & 0.039 & -0.053 & -0.041 & -0.053 & 1.000 & 1.000 \\
\hline & Sig.(bilateral) & 0.652 & 0.716 & 0.623 & 0.706 & 0.574 & 0.623 & \\
\hline & $\mathrm{N}$ & 88 & 88 & 88 & 88 & 88 & 88 & 88 \\
\hline
\end{tabular}

Tabla 3: Prueba de Kruskal-Wallis (variable agrupación AM GLOBAL)

\begin{tabular}{|c|c|c|c|c|c|c|}
\hline & Edad & Sexo & Ingeniería & $\begin{array}{c}\text { Ansiedad } \\
\text { Aprendizaje }\end{array}$ & $\begin{array}{c}\text { Ansiedad } \\
\text { Evaluación }\end{array}$ & Desempeño \\
\hline $\begin{array}{c}\text { Hde Kruskal- } \\
\text { Wallis }\end{array}$ & .317 & 3.051 & 22.818 & 18.591 & 88.000 & 1.911 \\
\hline gl & 2 & 2 & 2 & 2 & 2 & 2 \\
\hline Sig. asintótica & .854 & .218 & .000 & .000 & .000 & .385 \\
\hline
\end{tabular}




\section{DISCUSIÓN}

En el presente trabajo se ha realizado un estudio de la Ansiedad matemática y su asociación con el rendimiento académico y variables asociadas como tipo de ingeniería, edad y género. El análisis correlacional indica, en primera instancia, que no existe asociación entre la ansiedad matemática y el desempeño académico, tal y como dice el estudio de Krinzinger et al. (2009); esto se contrapone a otros estudios en los cuales se presenta una correlación moderada negativa (Ashcraft y Krause, 2007; Devine et al., 2012; Jansen et al., 2013), por lo que se puede decir que no hay una visión unánime al respecto, puesto que este fenómeno no se puede explicar de una forma tan sencilla ya que según Agüero et al.(2017), dicen que son diversos los factores que intervienen para ello, entre los que destaca el autoconcepto que se tiene acerca de la habilidad para las matemáticas, que hace repercutir tanto de forma positiva o negativa, en su rendimiento.

A su vez se ha considerado esencial para ser capaces de poder explicar la ausencia de correlación entre rendimiento académico y ansiedad, la variable temporal, esta variable hace referencia a las condiciones evaluativas bajo las cuales el alumnado se le impone un límite temporal para la resolución de estas pruebas, dando como resultado un rendimiento mucho menor que en evaluaciones donde no se establecen espacios temporales para su realización. La limitación temporal en este tipo de situaciones aumenta la ansiedad imposibilitando que el alumnado pueda desarrollar y plasmar todas sus competencias y habilidades matemáticas, resultando perjudicado su rendimiento (Ashcraft y Ridley, 2005; Tejedor et al., 2009).

Los resultados, que si muestra la tabla 1 del estudio, son la relación negativa entre la edad y la ingeniería, lo que explica que cuantos más años tienen los estudiantes menos eligen estudiar ingeniería, esto puede tener diversas causas, aunque la más plausible se debe a la dificultad de estos grados, los cuales requieren mucha dedicación para superarlas, y las personas de más edad precisamente no disponen de él debido a que tienen familia y trabajo a los que atender. Sin embargo, una de las asociaciones más importantes de este mismo, es la aparición de una correlación negativa entre la edad y la ansiedad a las matemáticas, de lo que se puede inferir que los estudiantes experimentan más ansiedad cuanto más jóvenes son, en consonancia con autores como Rahim y Koeslag (2005).

En otro sentido, se encuentra que existe una correlación entre la ingeniería y la ansiedad matemática global lo que puede relacionarse con el resultado anterior, ya que la mayoría de los estudiantes de ingeniería son jóvenes. Eso hace que a más estudiantes de ingeniería, haya un aumento proporcional de la ansiedad entre sus estudiantes, en consecuencia, la mayoría de los que seleccionen esos estudios tendrán menos años, por lo que sería altamente recomendable la creación de programas de retención con calidad de estudiantes en los estudios de ingeniería, promoviendo procesos de intervención que permitan un adecuado manejo de estrategias pedagógicas docentes y estrategias de aprendizaje en los estudiantes que favorezcan minimizar el riesgo de que esta pueda afectar el rendimiento en los estudiantes, con claros beneficios para todas las partes involucradas.

Con respecto al género no se han hallado diferencias significativas, aunque si bien es cierto que cuando se ha comprobado la media de la AM GLOBAL sí se han encontrado diferencias entre los hombres y mujeres siendo más pronunciada la ansiedad en ellas, en un 2 frente al 1,6 de ellos. Al igual que con la variable Rendimiento no hay un consenso en la comunidad científica sobre esta variabilidad, tal y como indican los trabajos de Ashcraft y Krause (2007), esas variables mientras que otros dicen que no hay diferencias estadísticamente significativas como para poder afirmar dicha relación, por lo que se necesitaría profundizar en este aspecto, para intentar hallar un consenso en el futuro. Por otra lado, el predominio de género masculino en un $65,9 \%$ de estudiantes de ingeniería podría estar relacionado de igual manera a aspectos sociales y culturales relacionados con estereotipos de género, entendiendo que los roles ocupacionales tienen un componente cultural relacionado con la función que éste le asigna a cada uno, es decir, los estudios relacionados con las matemáticas, se han considerado predominantemente masculinos (Del Río et al., 2016), por lo que concienciar a la población estudiantil desde sus inicios, se considera vital para conseguir que estos estereotipos no se perpetúen, como consecuencia, es importante dedicar esfuerzos investigativos en este sentido.

En cuanto al estadístico de Kruskal-Wallis que permite discernir la varianza que demuestra la ansiedad matemática en función de la ingeniería, la ansiedad en el aprendizaje y en la evaluación de las matemáticas. En esta prueba nos indica que hay diferencias significativas en la varianza de la ansiedad con respecto a la ingeniería elegida por los sujetos de la investigación, lo cual va en la línea de lo que indican los estudios de Furner y Berman (2004) o Pérez-Tyteca (2012). Estos estudios plantean que la ansiedad matemática es capaz de intervenir en la toma de decisiones a la hora de seleccionar áreas con una fuerte presencia de las matemáticas en su currículo, especialmente cuando éstas dejan de ser obligatorias en los currículos educativos. Tal y como argumentan Furner y Berman (2004) "a menudo los estudiantes ansiosos, aburridos, miedosos o que simplemente creen que las matemáticas no son importantes son propensos a evitar el estudio de la materia" ( $p .1)$ y que "los estudiantes deben superar sus miedos hacia las matemáticas ya que, si no lo hacen, éste se puede ver reflejado en la elección de su futura carrera, si ellos mismos no desafían a tomar cursos de matemáticas avanzadas" (p.7). 
Todo ello toma más relevancia cuando se observa cómo se distribuye esta ansiedad entre las 3 ingenieras en las que se distribuyen los participantes de la investigación, ya que forman parte de los cursos de Ingeniería Industrial, de Sistemas y de Mercados; en donde, en las Ingenierías de Sistemas e Industrial, la presencia de las matemáticas, toma un rol más importante.

En definitiva, la Ansiedad matemática es un constructo muy importante para el sistema educativo, el cual a través de los años ha sido y sigue siendo invisible, puesto que no se tienen en cuenta las consecuencias que esta puede ocasionar en el alumnado tanto a nivel fisiológico como a nivel psicológico y educativo, por eso sería muy adecuado tener en cuenta que los individuos que presentan una elevada ansiedad hacia las matemáticas, eluden los itinerarios en donde más entra en acción esta asignatura, y esto se traduce en un peor conocimiento de la materia, reduciendo o coartando su libertad a la hora de seleccionar estudios en los niveles superiores cuando esta materia deja de ser obligatoria y pasa a ser voluntaria. Se considera entonces que es importante desarrollar investigaciones en las que convergen estas tres variables: desempeño, ansiedad estado y autoconcepto; ya que en esta tríada es cuando se puede observar un aumento de la ansiedad matemática.

\section{CONCLUSIONES}

De acuerdo al trabajo presentado y a los resultados obtenidos se pueden plantear las siguientes conclusiones principales:

1.- Los resultados descriptivos muestran que los estudiantes presentan en un alto porcentaje de ansiedad moderada. Este comportamiento no es el deseado, ya que si un individuo escoge una carrera con una alta presencia de asignaturas relacionadas con las matemáticas, como es el caso de las ingenierías, se espera que su actitud y sus emociones hacia ellas, sean lo más positivas posibles, y la ansiedad hacia esta asignatura no debería existir.

2.- Realizando un análisis de la población objeto de estudio, se confirma la tendencia resultante frente al género femenino y su no preferencia hacia carreras que incluyan las matemáticas, lo que limita sus opciones a la hora de seleccionar los estudios que desea cursar, teniendo en mente la posibilidad de sufrir ansiedad, en el grado que sea.

3.- Por otro lado, el análisis de correlación realizado en esta investigación sugiere que entre la ansiedad matemática y el desempeño académico no existe; no obstante, si se hallan relaciones entre la edad y la ingeniería que estudian y entre la ansiedad matemática global y el tipo de ingeniería. De todo ello podemos concluir que la influencia del desempeño académico con respecto a la ansiedad no está claramente definida, el desempeño real de los investigados pudiera estar más influenciada por otros aspectos como podría ser su propio autoconcepto (Agüero et al., 2017), o una posible ansiedad estado.

Aunque ya ha quedado patente en la literatura de la corriente principal la importancia de estudiar la relación entre ansiedad matemática y el desempeño académico, ésta temática no ha sido suficientemente abordada en Colombia y en los países del entorno, por lo que creemos que es necesario llevar a cabo más estudios en estos países. También sería interesante diseñar estrategias encaminadas a reducir la ansiedad matemática, no solo en ingeniería, sino en todas las etapas educativas.

\section{AGRADECIMIENTOS}

Los autores agradecen a la Universidad Simón Bolívar Colombia con sede en Barranquilla, por facilitarnos los datos necesarios para su elaboración y su buen disponer durante todo el proceso.

\section{REFERENCIAS}

Agüero, E., Meza, L.G., Suárez, Z. y Schmidt, S., Estudio de la ansiedad matemática en la educación media costarricense, Revista Electrónica de Investigación Educativa, 19(1), 35-45 (2017).

Ashcraft, M.H. y Krause, J.A., Working memory, math performance and math anxiety, doi: 10.3758/BF03194059, Psychonomin Bulletin \& Review 14, 243-248 (2007).

Ashcraft, M.H. y Ridley, K.S., Cognitive consequences of math anxiety: A tutorial review., in Handbook of Mathematical Cognition, Campbell, J.I.D., pp. 315-327, Psychology Press, Hove, UK (2005).

Bauselas-Herreras, E., PISA 2012: Ansiedad y Bajo Rendimiento en Competencia Matemática, Revista Iberoamericana de Diagnóstico y Evaluación-e Avaliação Psicológica, 1(46), 161-173 (2018).

Brown, J.L. y Sifuentes, L.M., Validation study of the abbreviated math anxiety scale: Spanish adaptation, Journal of Curriculum and Teaching, 5(2), 76-82 (2016).

Carey, E., Hill, F., Devine, A. y Szücs, D., The chicken or the egg? The direction of the relationship between mathematics anxiety and mathematics performance, doi: 10.3389/fpsyg.2015.01987, Frontiers in Psychology, 6, 1-6 (2016). 
Cerda, G., Ortega, R. y otros tres autores, Predisposición desfavorable hacia el aprendizaje de las Matemáticas: una propuesta para su medición, Estudios Pedagógicos (Valdivia), 42(1), 53-63 (2016).

Cerda, G.A., Salcedo., P.A., Pérez, C.E. y Marín, V., Futuros Profesores de Matemáticas: Rol de la Disponibilidad Léxica, Esquemas de Razonamiento Formal en Logros Académicos Durante su Formación Inicial, doi: 10.4067/S071850062017000100005, Formación universitaria, 10(1), 33-46, (2017).

ICFES., National results report, Colombia in PISA 2015 Bogotá, Colombia: Author (2017).

Del Río, M.F., Strasser, K. y Susperreguy, M.I., ¿Son las habilidades matemáticas un asunto de género?: Los estereotipos de género acerca de las matemáticas en niños y niñas de Kínder, sus familias y educadoras, Calidad en la Educación, (45), 20-53 (2016).

Devine, A., Fawcett, K., Szücs, D. y Dowker, A., Gender differences in mathematics anxiety and the relation to mathematics performance while controlling for test anxiety, doi: 10.1186/1744-9081-8-33, Behavioral and Brain Functions, 8, 1-9 (2012).

Eccius-Wellmann, C., Lara-Barragán, A.G., Martschink, B. y Freitag, S., Comparison of mathematical anxiety profiles between Mexican students and German students, IberoAmerican Journal of Higher Education, 8(23), $69-83$ (2017).

Furner, J. M. y Berman, B.T., Building math confidence for a high-tech world, Academic Exchange Quarterly, 8(2), 214-221 (2004).

García-Santillán, A., Martínez-Rodríguez, V. y Santana, J.C., Psychometric Properties of the RMARS Scale in High School Students, European Journal of Contemporary Education, 7(1), 97-117 (2018).

Hopko, D.R., Mahadevan, R., Bare, R.L. y Hunt, M.K., The abbreviated math anxiety scale (AMAS): Construction, validity, and reliability. Assessment, 10(2), 178-182 (2003).

Isiksal, M., Curran, J.M., Koc, Y. y Askun, C.S., Mathematics anxiety and mathematical self-concept: Considerations in preparing elementary-school teachers, Social Behavior and Personality: An International Journal, 37(5), 631-643 (2009).

Justicia-Galiano, M.J., Pelegrina, S. y otros cuatro autores, Math anxiety and its relationship to inhibitory abilities and perceived emotional intelligence, Anales De Psicología/Annals of Psychology, 32(1), 125-131 (2016).

Jansen, B.R., Louwerse, J. y otros cuatro autores, The influence of experiencing success in math on math anxiety, perceived math competence, and math performance, doi: 10.1016/j.lindif.2012.12.014, Learning and Individual Differences, 24, 190-197 (2013).

Krinzinger, H., Kaufmann, L. y Willmes, K., Math anxiety and math ability in early primary school years, Journal of Psychoeducational Assessment, 27(3), 206-225 (2009).

Maloney, E.A., Schaeffer, M.W. y Beilock, S.L., Mathematics anxiety and stereotype threat: shared mechanisms, negative consequences and promising interventions, Research in Mathematics Education, 15(2), 115-128 (2013).

Maury, S.C., Marín, J.C., Ortiz, M. y Gravini, M., Competencias genéricas en estudiantes de educación superior de una universidad privada de Barranquilla Colombia, desde la perspectiva del Proyecto Alfa Tuning América Latina y del Ministerio de Educación Nacional de Colombia (MEN), Revista Espacios, 39(15) (2018).

OECD., PISA 2018 Results (Volume l): What Students Know and Can Do, PISA, doi: 10.1787/5f07c754-en, OECD Publishing, Paris, pp. 133 (2019).

O'Leary, K., Fitzpatrick, C.L. y Hallett, D., Math anxiety is related to some, but not all, experiences with math, doi: 10.3389/fpsyg.2017.02067, Frontiers in Psychology, 8(2067), 1-14 (2017).

Pérez-Tyteca, P., La ansiedad matemática como centro de un modelo casual predictivo de la elección de carreras, (Tesis doctoral), Universidad de Granada, (2012).

Pérez-Tyteca, P., Parrilla, J.M. y Martínez, E.C., Afecto y matemáticas. Diseño de una entrevista para acceder a los sentimientos de alumnos adolescentes, Avances de Investigación en Educación Matemática, (4), 65-82 (2013).

Pérez-Tyteca, P., Martínez, E.C., Romero, L.R. y Martínez, E.C., Ansiedad matemática, género y ramas de conocimiento en alumnos universitarios. Enseñanza de las Ciencias: Revista de Investigación y Experiencias Didácticas, 29(2), 237-250 (2011).

Rahim, M. y Koeslag, M., Math anxiety in Junior/Intermediate preservice teachers, Alan Regerson, Reform, Revolution and Paradigm shifts in Mathematics Education, 194-198 (2005).

Reali, F., Jimenez-Leal, W. y otros tres autores, Examining the link between mathematical anxiety and mathematical performance in Colombian students, doi: 10.15446/rcp. v25n 2.54532, Revista Colombiana de Psicologia, 25(2), 369-379 (2016).

Rodríguez-Ayán, M. N., La perspectiva estudiantil sobre el desempeño del profesor: un modelo de regresión logística ordinal, https://doi.org/10.17811/rema.10.1.2005.1-13, Revista Electrónica de Metodología Aplicada, 10(1), 1-13 (2005).

Tejedor, B., Santos, M.A. y otros tres autores, Variables explicativas de la ansiedad frente a las matemáticas: un estudio de una muestra de 6o de primaria, Anuario de Psicología, 40(3), 345-355 (2009). 